\title{
Prevalence of Intrinsic and Extrinsic Factors Contributing to Causing of Functional Decline in Elderly Individuals in the Rural Population of Karad, India
}

\author{
Urwashi Kamal Kant Baurai ${ }^{1}$, Malti Vijay Bhambure ${ }^{2}$, Anandh Srinivasana ${ }^{3}$ \\ 1, 2 Department of Physiotherapy, Krishna Institute of Medical Sciences (Deemed to Be University), \\ Malkapur, Karad, Maharashtra, India, ${ }^{3}$ Department of Community Health Sciences, Krishna Institute of \\ Medical Sciences (Deemed to Be University), Malkapur, Karad, Maharashtra, India.
}

\section{ABSTRACT}

\section{BACKGROUND}

Functional decline refers to increase in dependence towards activities of daily living and is common with increasing age, chronic diseases, and previous hospitalization. We wanted to determine the rate of intrinsic and extrinsic factors of functional decline in different age groups among elderly population and in relation to gender.

\section{METHODS}

The study population was composed of 64 individuals which were above the age group of 60 years, further subdivided into 3 groups: 60 - 70, 70 - 80 and 80 years and above. The validated questionnaire was provided through which the intrinsic and extrinsic factors were determined.

\section{RESULTS}

In all the studied subjects, extrinsic factors had a mean value of $31.24 \%$ while intrinsic factors had a mean value of $28.12 \%$ which indicates that extrinsic factors are more prevalent than intrinsic factors in all the age groups. Both intrinsic and extrinsic factors were more prevalent in the female population than the male population with the prevalence rate of intrinsic factors as $34.37 \%$ in males and $50 \%$ in females and for the extrinsic factors prevalence rate of $39.06 \%$ in males and 54.68 $\%$ in females. In age group of $60-70$ yrs. we found that the prevalence of intrinsic factors was (23.43\%) and extrinsic was (26.56\%), for 70 - 80 intrinsic it was (29.68 $\%)$ extrinsic was (32.81\%) and for 80 and above prevalence intrinsic was (31.25\%) and extrinsic was (34.37\%).

\section{CONCLUSIONS}

The study showed that the extrinsic factors contributed more to the functional decline as compared to the intrinsic factors. These findings are important as the functional decline and increasing care needs are potentially modifiable.

\section{KEY WORDS}

Functional Decline, Intrinsic Factors, Extrinsic Factors, Disability
Corresponding Author:

Dr. S. Anandh,

Krishna Institute of Medical

Sciences (Deemed to Be

University), Malkapur, Karad,

Maharashtra, India

E-mail: anandh73@gmail.com

DOI: $10.14260 / j e m d s / 2021 / 551$

How to Cite This Article:

Baurai UKK, Bhambure MV, Srinivasana A. Prevalence of intrinsic and extrinsic factors contributing to causing of functional decline in elderly individuals in the rural population of Wardha, India. J Evolution Med Dent Sci 2021;10(33):2697-2701, DOI: $10.14260 /$ jemds $/ 2021 / 551$

Submission 08-04-2021, Peer Review 13-06-2021 Acceptance 19-06-2021, Published 16-08-2021.

Copyright (c) 2021 Urwashi Kamal Kant Baurai et al. This is an open access article distributed under Creative Commons Attribution License [Attribution 4.0 International (CC BY 4.0)] 


\section{BACKGROUND}

Functional decline is a dependency in the self-care capabilities and is identified by the depreciation in mobility and in the performance of daily living activities such as dressing, toileting and bathing. Functional decline becomes evident with ageing and is common in the older adults. It occurs in episodes and is associated with the subsequent health decline. There are various factors associated with functional decline - Intrinsic factors which are related to human body such as age, gender, chronic diseases, and the balance disorders. Extrinsic factors which involve the physical and socio-economic environment. Another factor which comes with aging is the functional decline which affects the activities of daily living (ADL) of the individuals. Activities of daily living are those activities that are essential for living on a daily basis which include self-care tasks such as eating, bathing, dressing, grooming, walking, and transferring from chair to bed and continence ${ }^{1}$ which can be assessed using (BARTHEL INDEX).

Functional decline leads to disability. Disability is associated with high mortality rates which leads to adverse outcomes such as hospitalization, admission in nursing homes etc. Therefore, early detection and prevention of risk factors can lead to reductions in the physical, emotional, social, and financial problems attributable to disability and improve the quality of life. ${ }^{2}$ Many studies have shown that the older adults have lower quality of life (QOL), with more diseases and greater functional declines. ${ }^{3}$ Functional decline is associated with high risk of subsequent health decline. The severity of limitation in physical functioning and mobility is determined by physical impairments which may be caused by either medical conditions or due to the external factors such as social support, financial support and environment. When multiple health issues are present, they often result in greater disability than expected because the patient's ability to compensate for one problem may be affected by comorbid conditions. ${ }^{4}$

Common health conditions that may contribute to functional decline may include cardiorespiratory diseases, neurological conditions, cancer, obesity, dementia, affective disorders, ophthalmic and auditory disorders and fractures. About 463 to 374 million adults worldwide face major health challenges such as Type 2 diabetes and prediabetes which are associated with the functional decline. ${ }^{5}$ The individuals having diabetes mellitus (Type 2) were thus included in this study. There are other diseases which are less disabling, but are common (e.g. arthritis). 6 The disabling effects vary, it depends upon the task the patient is trying to perform; for example, cardiovascular disease is more likely to cause difficulty in tasks that demand high aerobic work (e.g., housework), whereas neurological condition such as stroke is more likely to cause difficulty with basic self-care tasks that require limb mobility. ${ }^{7}$

Factors that contribute in causing functional decline are the coexistence of two or more health conditions that create more disability than expected. As the number of impairments rises, the percentage of persons reporting the functional dependence increases enormously. ${ }^{8}$ Such interconnection with the impairments may occur due to the interchange of the normal physiologic compensatory strategies. For example, a condition that reduces biomechanical performance of muscles and joints (e.g., arthritis) can increase the work of walking, and a patient with concomitant heart disease may lack the capacity to compensate increased demand.9,10 Another factor associated with the functional decline is hospitalization. Hospitalization is a stressful event in older people.11 Previous studies have shown that about $1 / 3^{\text {rd }}$ of the elderly population develop functional decline following hospitalisation. ${ }^{12,13}$ It is estimated according to the previous study that the rate of hospitalization usually increases at the age of 65 or older and as the age advances. ${ }^{14}$ There are still considerable number of environmental risk factors which can potentially be modified in the acute hospital setting such as avoiding prolonged bedrest. ${ }^{11}$ It is therefore important to target this group of the population for early physical therapy so as to minimize prolonged bed rest and deconditioning which may result in new problems contributing to the functional decline.

There is enormous need to assess the factors that may lead to the functional decline. Moreover, there is scanty research done in this area regarding the environmental risk factors. In this study the environmental and the social risk factors of the functional decline have been focused on. Thus, the present study was conducted to determine the rate of intrinsic and extrinsic factors of functional decline in different age groups among elderly population and in relation to gender.

\section{METHODS}

An institutional protocol committee was set up which verified the project study and later an ethical clearance was obtained by the Institutional Ethics committee of Krishna Institute of Medical Sciences, Deemed to Be University, Karad, to proceed with the study.

It was an observational study conducted among elderly population above 60 years of age in the rural population after taking informed consent. The study lasted for 6 months which was conducted from September 2019 to February 2020. It was a short-term study investigating the factors associated with the functional decline in the elderly population of Karad. This study included the subjects who were severely or moderately dependent based on Barthel index, previously hospitalized 5 10 years, who used assistive devices, those who had fall in the last one year, and the ones who had chronic diseases among any of the following: Hypertension, Diabetes mellitus, Arthritis. The dependence of the individuals is measured by Barthel Index i.e., the final score on the Barthel index is multiplied by 5 and scored on a 100 - point scale where, 0 - 20: indicates total dependency, 21 - 60: severe dependency, 61 90: moderate dependency and 91 - 99: slight dependency. After this a self-made, validated questionnaire was given to the participants to determine the intrinsic and the extrinsic factors which were associated with the functional decline in the rural population. ${ }^{15}$ The questionnaire was prepared in English language originally and was then translated to local language for data collection. The questionnaire consisted of 2 parts: A] Restrictive Intrinsic factors toward functional decline and $B]$ Restrictive extrinsic factors toward functional decline. The first part of questionnaire consisted of 3 components which had 9 questions in total. The second part consisted of 13 questions.

The intrinsic factors were age, chronic disease, eyesight, hearing, health perception, fracture after 50 years of age, musculoskeletal problems, drugs and frailty index. The extrinsic factors included in the questionnaire were type of 
housing, electricity status, lighting in the house, number of stairs, type of floor, presence of handrails, living status, footwear, construction material in the house, assistive device used, type of toilet, previous hospitalization, habits like tobacco chewing and smoking.

Among a population of 200 people, we did simple random sampling using lottery method. We assigned a unique number to each participant and mixed them thoroughly in a bowl. Then one blind folded researcher among us selected 131 candidates. The sample size was calculated using formula: $\mathrm{n}=4 \mathrm{pq} / \mathrm{L}^{2}$, where $p$ indicates prevalence of functional decline $=20.5 \%, 16$ $\mathrm{q}=100-\mathrm{p}(79.9 \%), \mathrm{L}=$ allowable error $=10 \%$. According to the above formula, we got a sample size of 64 .

We had to exclude 50 patients as they did not fit in the inclusion criteria; 8 had severe psychiatric disorders, 29 were bedridden for a long period and 13 candidates declined to participate. Out of this remaining population of 81 candidates (130 - 50), the study included the individuals who were aged 60 years \& above. They were further categorized in to the three sub groups of 60 - 70 years, 70 - 80 years and 80 years \& above. We had to narrow down the study population to 64 according to the calculated sample size. For obtaining this, we again performed simple random sampling by lottery method and selected 64 candidates. On recruitment the patients received a patient information sheet and signed the consent form. All the candidates who were willing to participate in the study filled the questionnaire.

\section{Statistical Analysis and Interpretation}

The questionnaire filled by every individual was assessed thoroughly. Each option in every question was designated a number, example: option $A$ was given 1 and option $B$ was given 2. We counted the number attributed to each option and calculated the percentage for each option. Each option signifies different factors associated for functional decline. To check if there is any co-relation between the 2 factors, chi square test was done.

\section{RESULTS}

In all the studied subjects, extrinsic factors had a mean value of $31.24 \%$ while intrinsic factors had a mean value of $28.12 \%$ which indicates that extrinsic factors are more prevalent than intrinsic factors in all the age groups. As the age group of the patient increases, the prevalence of factors also increases.

In the male and female population, the prevalence of intrinsic factors was $34.37 \%$ and $50 \%$ respectively. In the male and female populations, the prevalence of extrinsic factors was $39.06 \%$ and $54.68 \%$ respectively.

Among the age group of $60-70 \mathrm{yrs}$. we found that the prevalence of intrinsic factors was $(23.43 \%)$ and extrinsic was (26.56\%), for 70 - 80 intrinsic it was (29.68\%) extrinsic was $(32.81 \%)$ and for 80 and above prevalence intrinsic was $(31.25 \%)$ and extrinsic was (34.37\%). Hence the present study shows that the rate of factors associated with functional decline increases as the age advances.

In a total of 21 patients with lower socio-economic status, 18 patients had good health and 3 had bad health according to the questionnaire. Chi square tests were done to find out any correlation between the factors but as the $\mathrm{P}$ value came out to be $>0.05$, there was no correlation.

\begin{tabular}{|cccc|}
\hline Class ( Socio-Economic Status) & A & B & Total \\
Lower & 18 & 3 & $\mathbf{2 1}$ \\
Middle & 30 & 13 & $\mathbf{4 3}$ \\
\hline Table 1. Health Status & & \\
\hline Chi square- 1.914, P Value- 0.1666 & \\
\hline
\end{tabular}

\begin{tabular}{|cccc|}
\hline Living Status & A & B & Total \\
Alone & 8 & 5 & $\mathbf{1 3}$ \\
With Family & 30 & 13 & $\mathbf{4 3}$ \\
\hline \multicolumn{4}{c|}{ Table 2. Health Status } \\
\hline Chi Square $-1.577, \mathrm{P}$ Value & -0.2092 \\
\hline
\end{tabular}

In a total of 13 patients who were living alone, 8 patients had good health and 5 had bad health according to the questionnaire. Among 51 people who were living with family, 40 had good health and 11 had bad health according to the questionnaire. Chi square tests were done to find out any correlation between the factors but as the $\mathrm{P}$ value was $>0.05$, there was no correlation.

\begin{tabular}{|ccccc|}
\hline Gender & A & B & C & Total \\
Males & 0 & 18 & 8 & 26 \\
Females & 1 & 21 & 16 & 38 \\
\hline \multicolumn{5}{|c|}{ Table 3. Frailty Index } \\
\hline Chi Square- 1.707, P Value- 0.4258 \\
\hline
\end{tabular}

In a total of 26 male patients, 18 patients had frailty risk and 8 had frailty according to the questionnaire. Among 38 female patients, 1 had no frailty and 21 had frailty risk and 16 had frailty according to the questionnaire. Chi square tests were done to find out any relation between the factors but as the $\mathrm{P}$ value was $>0.05$, there was no correlation.

\begin{tabular}{|cccccccc|}
\hline Frailty Index & A & A, B & A, C & B & B, C & C & Total \\
No frailty & 0 & 0 & 0 & 0 & 0 & 1 & 1 \\
Frailty risk & 9 & 4 & 1 & 17 & 3 & 5 & $\mathbf{3 9}$ \\
Frailty & 5 & 3 & 1 & 8 & 4 & 3 & $\mathbf{2 4}$ \\
\hline \multicolumn{10}{c}{ Table 4. Chronic Diseases } \\
\hline Chi Square- 7.928, P Value- 0.6359
\end{tabular}

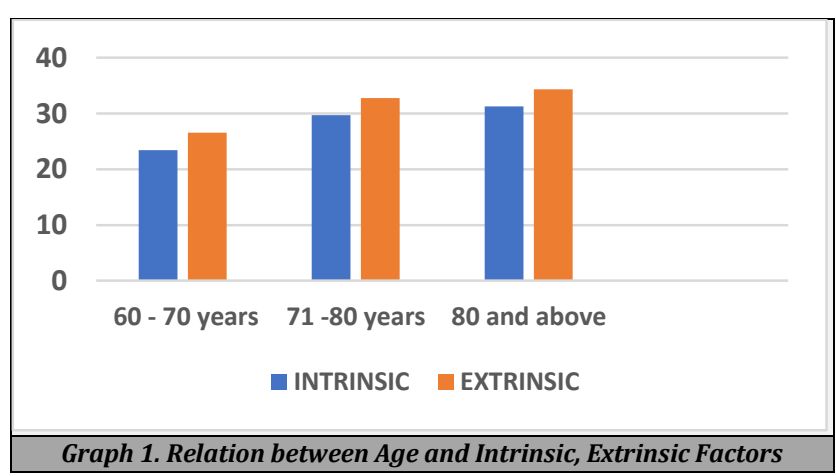

In the questionnaire, 3 chronic diseases were included. Option A was Arthritis, Option B was Hypertension and C was Diabetes mellitus. Chi square tests were done to find out any correlation between the factors but as the $\mathrm{P}$ value came out to be $>0.05$, there was no correlation.

\section{P Value}

1. Health status vs Socio economic status 0.1666 (Table 1)

2. Health status vs living status 0.2092 (Table 2)

3. Frailty index vs gender 0.4258 (Table 3 )

4. Chronic diseases vs Frailty index 0.6359 (Table 4) 
As the P values were greater than 0.05 ( $>0.05$ ), it was not statistically significant. Therefore, there was no co-relation between the above-mentioned components.

Graphical representation of the data showed pictorial description of the factors that were dominantly contributing in the functional decline of the selected population. (Refer Graph 1).

\section{DISCUSSION}

By the year 2050, India will have 34 crore people above 60 years of age which forms a huge part of population and their health cannot be ignored. One of the problems which is encountered with increasing age is functional decline which is associated with disability and high mortality rates. ${ }^{14}$ In the previous studies, various factors which are responsible for the functional decline have been found out but extensive focus on extrinsic factors was not done.

There are gaps and conflicting findings in the relative importance on various risk factors and the best ways of intervening environmental risk factors have largely been neglected in earlier research, which needs to be broadly focused on. ${ }^{13}$ Environmental setup, public home settings show variations in India where there is a need for regional survey.

As functional decline is common in the elderly population there is even restriction of the activities of daily living which may hamper their quality of life. And determining the rate of factors that can contribute in causing the functional decline will help to intervene earlier according to the cause.

This study was carried out on a sample of 64 elderly subjects aged over 60 years. The population was further subdivided into 3 age groups i.e., 60 - 70, 70 - 80, 80 and above, this was done to assess the rate and the factors associated with the functional decline with the increasing age. The subjects were assessed by the terms of Barthel index and those who were moderately and severely dependent according to this scale were further taken for the study. The individuals were provided a questionnaire for the determination of the intrinsic and extrinsic factors and general demographic data was collected. While comparing this study with the research previously done by Inaki Martin Lesende, Luis Ignacio Mendibil Crespo, Sonia Castano Manzanares et al. (2018) which explained about the functional decline and associated factors related to multimorbidity but very few studies were done to determine the prevalence of the intrinsic as well as extrinsic factors in elderly individuals.

In this study health perception factors included the overall general health, vision, hearing, presence of the chronic diseases such as hypertension, diabetes mellitus, arthritis. Economic factors such as socio-economic status were determined.

The other restrictive intrinsic factors such as musculoskeletal problems, frailty index, medications, duration of medications were the factors that were ascertained based on the positive response of the question. While the restrictive extrinsic factors included the questions like the type of housing, lightning in the house, floors are slippery / even or uneven, stairs at home do they have handrails, toilet - Indian or commode, electricity status at home, footwear, assistive device, physical activities, previous hospitalization and habits such as smoking, alcohol, Misheri were determined using the questionnaire which was designed according to the factors which may affect functional decline in the rural population. The present study evaluates the rate of risk factors at early stage which will help to determine which factor influences more in causing functional decline, which will help in providing early intervention strategies which will eventually lead to decrease in the rate of functional decline and thus improvising the quality of life of the elderly individuals aged between 60 and 80 yrs.

Key features of this study that makes it important are it involves patients who were previously hospitalized. Another feature is that it is one of the few studies that focuses on the prevalence of the intrinsic as well as the extrinsic factors related to functional decline.

\section{CONCLUSIONS}

Functional decline is considered as one of the major concerns in the elderly population which leads to restriction in the activities of daily living. Our findings indicate that the functional decline is common among all the age groups of elderly population. And as per our findings we conclude that the rate of functional decline increases as the age advances. The factors contributing to the functional decline also worsens the health as the age advances. Among the 64 samples in the age group of $60-70$ yrs. we found that the prevalence of intrinsic factors was (23.43\%) and extrinsic was (26.56\%),for 70 - 80 intrinsic it was (29.68\%) extrinsic was $(32.81 \%)$ and for 80 and above prevalence intrinsic was (31.25\%) and extrinsic was (34.37\%)(refer Graph 1). Hence, the present study shows that the rate of factors associated with the functional decline increases as the age advances and the prevalence rate of the restrictive extrinsic factors that leads to the functional decline is more as compared to the rate of the restrictive intrinsic factors.

Therefore, it is necessary to improvise the environmental restrictive extrinsic factors by wearing suitable footwear, providing handrails on staircases for support, lifestyle modifications such as increasing the level of physical activities, levelling off the floor and improvising the lighting. Further, explorative study is required with larger sample size and diverse population so that early interventions can be done and can help prevent further disability.

\section{Limitations}

There are a few limitations to this study -

- The study was confined to a small number of subjects, which resulted in reduced power in statistical analysis.

- No standardized questionnaire was available; therefore, investigator prepared a questionnaire for the purpose of this study.

Data sharing statement provided by the authors is available with the full text of this article at jemds.com.

Financial or other competing interests: None.

Disclosure forms provided by the authors are available with the full text of this article at jemds.com. 


\section{REFERENCES}

[1] Wilber ST, Blanda M, Gerson LW. Does functional decline prompt emergency department visits and admission in older patients? Acad Emerg Med 2006;13(6):680-2.

[2] Gill TM, Baker DI, Gottschalk M, et al. A program to prevent functional decline in physically frail, elderly persons who live at home. $\mathrm{N}$ Engl J Med 2002;347(14):1068-74.

[3] Wijk H, Corazzini K, Kjellberg IL, et al. Person-cantered incontinence care in residential care facilities for older adults with cognitive decline: feasibility and preliminary effects on quality of life and quality of care. J Gerontol Nurs 2018;44(11):10-9.

[4] Colón-Emeric CS, Whitson HE, Pavon J, et al. Functional decline in older adults. Am Fam Physician 2013;88(6):388-94.

[5] Brown RT, Diaz-Ramirez LG, Boscardin WJ, et al. Functional impairment and decline in middle age: a cohort study. Ann Intern Med 2017;167(11):761-8.

[6] Verbrugge LM, Patrick DL. Seven chronic conditions: their impact on US adults' activity levels and use of medical services. Am J Public Health 1995;85(2):173-82.

[7] Ettinger WH Jr, Fried LP, Harris T, et al. Self-reported causes of physical disability in older people: the cardiovascular health study. J Am Geriatr Soc 1994;42(10):1035-44.

[8] Tinetti ME, Inouye SK, Gill TM, et al. Shared risk factors for falls, incontinence and functional dependence: unifying the approach to geriatric syndromes. JAMA 1995;273(17):1348-53.
[9] Wu YJ, Chen SY, Lin MC, et al. Energy expenditure of wheeling amputations. Arch Phys Med Rehabil 2001;82(2):265-9.

[10] Lapointe R, Lajoie Y, Serresse O, et al. Functional community ambulation requirements in incomplete spinal cord injured subjects. Spinal Cord 2001;39(6):32735.

[11] Jahan AM. The most important considerations in the assessment of functional decline in seniors: a literature review. Asian Pacific J Health Sciences 2017;4(1):61-70.

[12] WHO. Regional Office for Europe's Health evIdence Network. What are main risk factors for disability in old age and how can disability be prevented? September 2003.

[13] Manton KG. A longitudinal study of functional change and mortality in the United States. J Gerontol 1988;43(5):S153-61.

[14] Van Grootven B, Jeuris A, Jonckers M, et al. Predicting hospitalisation-associated functional decline in older patients admitted to a cardiac care unit with cardiovascular disease: a prospective cohort study. BMC Geriatr 2020;20(1):1-7.

[15] Staff A. The (Original) barthel index of ADLs. Elite Learning 2008. https://www.elitecme.com/resourcecenter/rehabilitation-therapy/the-original-barthelindex-of-adls/(last accessed 30.4.2019)

[16] Lesende IM, Crespo LIM, Manzanares SC, et al. Functional decline and associated factors in patients with multimorbidity at 8 months of follow-up in primary care: the functionality in pluripathological patients (FUNCIPLUR) longitudinal descriptive study. BMJ Open 2018;8(7):e022377. 